\title{
Design and Implementation of Path Establishment and Maintenance Technique for MANETs
}

\author{
Saikat Jana \\ Software Consultant \\ Surflex Technology Pvt Itd \\ Kolkata, India
}

\author{
Jayashree Singha \\ Computer Sc. \& Engg. \\ Siliguri Institute of Technology \\ Siliguri, India
}

\author{
Souvik Singha \\ Computer Sc. \& Engg. \\ Techno India University \\ Kolkata, India
}

\begin{abstract}
Mobile Ad hoc Network (MANET) is a collection of wireless mobile nodes that dynamically form a network temporarily without any support of central management. Moreover, every node in MANET moves arbitrarily making the multi-hop network topology to change randomly at uncertain times. In this paper proposed a Path Establishment \& Maintenance Algorithm (PE\&MA) that optimizes the established routes when feasible without incurring extra overhead. By utilizing promiscuous mode of operation, sender node, the border neighbor node within each zone to forward a packet, hop counts embedded in IP optional header of data packets to find shorter paths. PE\&MA avoid unnecessary aggressive and ephemeral route updates, which improves the protocol performance. Routing path optimality is important as optimal path reduces the packet drop ratio, end-to-end delay, and reduces energy dissipation of end-to-end data transmission. It will also be easier to secure multicast routing. PE\&MA for ZRP fits well for MANETs where bandwidth is limited, topology changes frequently, power is constrained and security problem is serious. Simulation results are presented to support our claim. In this paper the result based on metrics such as throughput, packet delivery ratio and average end-toend delay by using the NS-2.31 simulator.
\end{abstract}

\section{Keywords}

MANET, AODV, Nodes, Wireless, Delay

\section{INTRODUCTION}

In the next generation of wireless communication systems, there will be a need for the rapid deployment of independent mobile users. Significant examples include establishing survivable, efficient, dynamic communication for emergency/rescue operations, disaster relief efforts, and military networks. Such network scenarios cannot rely on centralized and organized connectivity and can be conceived as applications of mobile ad hoc networks (MANETs). A MANET is an autonomous collection of mobile users that communicate over relatively bandwidth-constrained wireless links. Because the nodes are mobile, the network topology may change rapidly and unpredictably over time [1]. The network is decentralized; all network activity, including discovering the topology and delivering messages, must be executed by the nodes themselves; that is, routing functionality will be incorporated into mobile nodes $[2,3]$.

\section{OVERVIEW ON PATH ESTABLISHMENT AND MAINTENANCE ALGORITHM}

In particular, nodes only maintain "active" routes between source and destination. To establish a route towards destination, a source node initiates a restricted flooding generally called route request (RREQ). The destination may receive multiple RREQ ${ }^{\text {ce }} \mathrm{s}$. However, it selects a route based on the shortest distance (hop-count) and sends a route reply (RREP) back to the source over the selected path. Path found during the route establishment phase (RREQ/RREP) are typically the shortest routes. But the established path may become suboptimal over time due to node mobility and lack of global topological information shorter routes make good impact on the performance of routing protocols. Routes with optimal hops (shorter paths) are less likely to break than routes with sub-optical paths. As a result of sub-optimal paths [3, 4, 5]. So nodes dissipate more energy due to more transmissions and the packet delivery rate of the protocol increases [6,7].

\subsection{Path Establishment Phase}

Here assuming that the source node is centered of the monitoring region and also assuming that the monitoring region is divided into three fixed zone like zone1, zone2 and zone3 each of degree 120 depending upon the radio coverage area of the source node. In proposed method, the PEA is called three times for three zones. In this algorithm it is considered that each zone has $\mathrm{n}$ number of nodes. In mobile ad hoc network the frequency, wavelength and the Processing Time (TP) and also Broadcast Time (BT) of all nodes $[8,9]$. In this algorithm the maximum distance of a node at each zone is calculated first and the IP address of maximum distance node at each zone are stored in an array. The Receiving Time (RT) of all nodes is different which is taken as input for the algorithm. After that the Transceiver Time (TT) for all nodes in the monitoring region is calculated. The distance of all nodes of monitoring region is calculated and the IP address of all that nodes are stored. Then the maximum distance of node at each zone is found out. The IP address of maximum distance node is copied into an array afterwards. The value of IP address of maximum distance node is returned. Using the algorithm the IP-address for the forwarding node in each zone are found out and the packet are forwarded. In figure 1 node $\mathrm{S}$ wants to establish a path for transmitting data towards the node $\mathrm{D}$. node $\mathrm{S}$ forward the hello message towards node $\mathrm{N} 1$ in zone1, node $\mathrm{N} 2$ in zone2 and node $\mathrm{N} 3$ in zone3 rather than all the neighbor nodes. After receiving the message border node within each zone again border node call the PEA to forward the message. Using this way the path is established in between the sender node $S$ and the receiving node $\mathrm{D}$. 


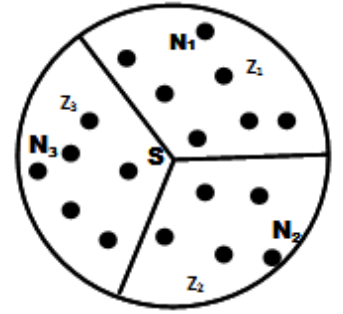

Fig 1: Working principle of PEA

The concept of dividing the radio cover age area into three zones and border nod e s election within each node can be used in enhancement of the proposed implementation of the Path Establishment Algorithm in the following way: The proposed implementation of the PEA requires that three arrays, say RT needs to be store the receiving time, say TT to calculate the transceiver time and say D, to store the distance for each node within each zone, These arrays in the recombination process at the receiver by providing the part numbers for subsequent recombination. Hence further processing can be done on this array before sending to enhance the effectiveness. This can be done through the concept of zone. Here, freq indicate the transmission power for the sender $[10,11,12]$.

The function will call three times for three $\operatorname{zone}\left(\mathrm{Z}_{1}, \mathrm{Z}_{2} \mathrm{Z}_{3}\right)$ and the value of IP1 should return to the program from where it is called and after getting the IP address sender node should forward the packet towards the node within zone. Same process will be follow for each receiving node until a path is established in between sender $\mathrm{S}$ and receiver $\mathrm{D}$. The proposed path is shown bellow.

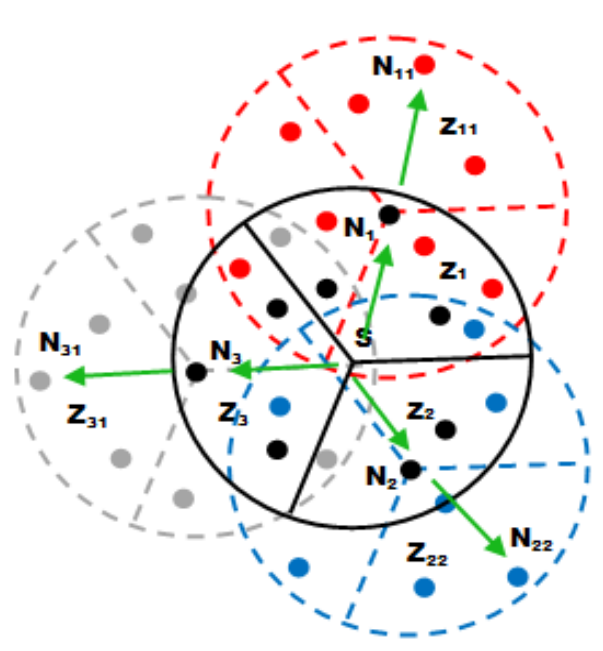

D

Fig 2: Path establishment using working principle of PEA

In the above PEA algorithm it is seen that if PEA is established on any source node (starting node), its select 3 nodes in its zone which contains of maximum distance from the source node. After every time it finds the 3 nodes, the PEA algorithm will be called for each of 3 nodes.

So that to reach the destination node from the source node. Each time the PEA algorithm will be run for "the power of three" times depending upon the steps it needs to cover the whole path. $1+3+3^{2}+\ldots .+3^{\mathrm{n}}$ times for the above case. Suppose 4 step it occurs for sending a node from source to destination, the PEA algorithm will be called for $1+3+3^{2}+3^{3}=40$ times. But for the proposed algorithm if after every time of PEA it can discard 2 of these nodes (show in figure 1.2) for hop and takes 1 node for minimum hop count.

For this algorithm, PEA will be called only for $\mathrm{n}$ times if it occurs $\mathrm{n}$ step to reach the destination. For the above case PEA algorithm will call for one means for the above case AODV will call for $n$ time. For the above example PEA will be called only $1+1+1+1=4$ time for four steps.

So here it is seen that the overhead is more less then the PEA.

\subsection{Path Maintenance Phase}

In proposed method the PEA is called three times for three zones. In this algorithm it is considered that each zone has $\mathrm{n}$ number of nodes. In mobile ad hoc network the frequency, wavelength and the Processing Time (TP) and also Broadcast Time (BT) of all nodes are different. In this algorithm the maximum distance of a node at each zone is calculated and the IP address of maximum distance node at each zone is stored in an array. The Receiving Time (RT) of all nodes is different which is taken as input for the algorithm. After that the Transceiver Time (TT) is calculated for all nodes in the monitoring region. Then the distance of all nodes of monitoring region is calculated and IP address of all that nodes are stored. Then the maximum distance of node at each zone are found. Then copy the IP address of maximum distance node into an array. Return the value of IP address of maximum distance node. After getting maximum distance node the destination is found using AODV flooding concept. AODV determines a root to destination only. Routs are maintained as long as they need by source. Using this, the hop count of each zone's maximum distance to destination is found. Then different hops are compared to set the minimum hop and the value of maximum distance node is returned. After ccompleting this step the PEA is called again to forward the packet. Using the algorithm the IPaddress for the forwarding node is found in each zone and forward the packet $[13,14]$. In figure 3 node $S$ wants to establish a path for transmitting data towards the node D. node $\mathrm{S}$ forward the hello message towards node $\mathrm{N} 1$ in zone1, node $\mathrm{N} 2$ in zone 2 and node N3 in zone 3 rather than all the neighbor nodes. After receiving the message border node within each zone, here count the hop to reach destination and stored the value in an array. Then compare hop each zone max distance node to destination node and set minimum hop. Then forward the packet min hop zone max distance node then again call the PEA to forward the message. Using this way the path is established in between the sender node $\mathrm{S}$ and the receiving node D.

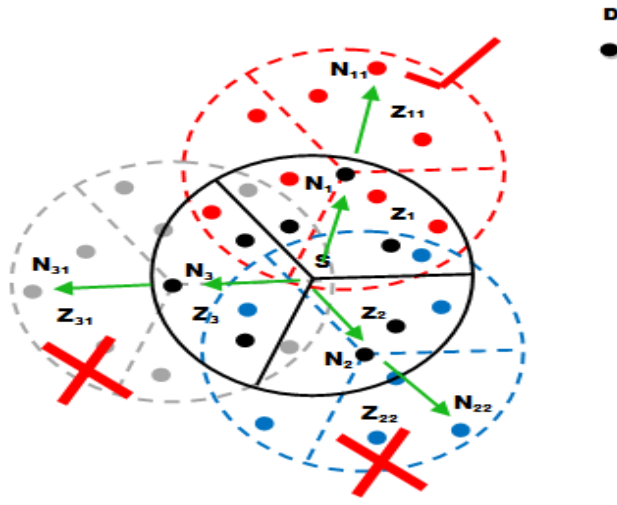

Fig 3 : set the zone using minimum hop count from source to destination

In this Figure 3 the distance $N_{1}$ to $D, N_{2}$ To $D$, and $N_{3}$ to $D$ are found using Hop count Then set the minimum Hop which is 
$\mathrm{N}_{1}$ to $\mathrm{D}$ in zone $\mathrm{Z}_{1}$ and discard $\mathrm{Zone} \mathrm{Z}_{2}$ and $\mathrm{Z}_{3}$. For this algorithm, PEA will be called only for $n$ times if it occurs $n$ step to reach the destination. For the above case PEA algorithm will called for one means for the above case AODV will call for $\mathrm{n}$ time.

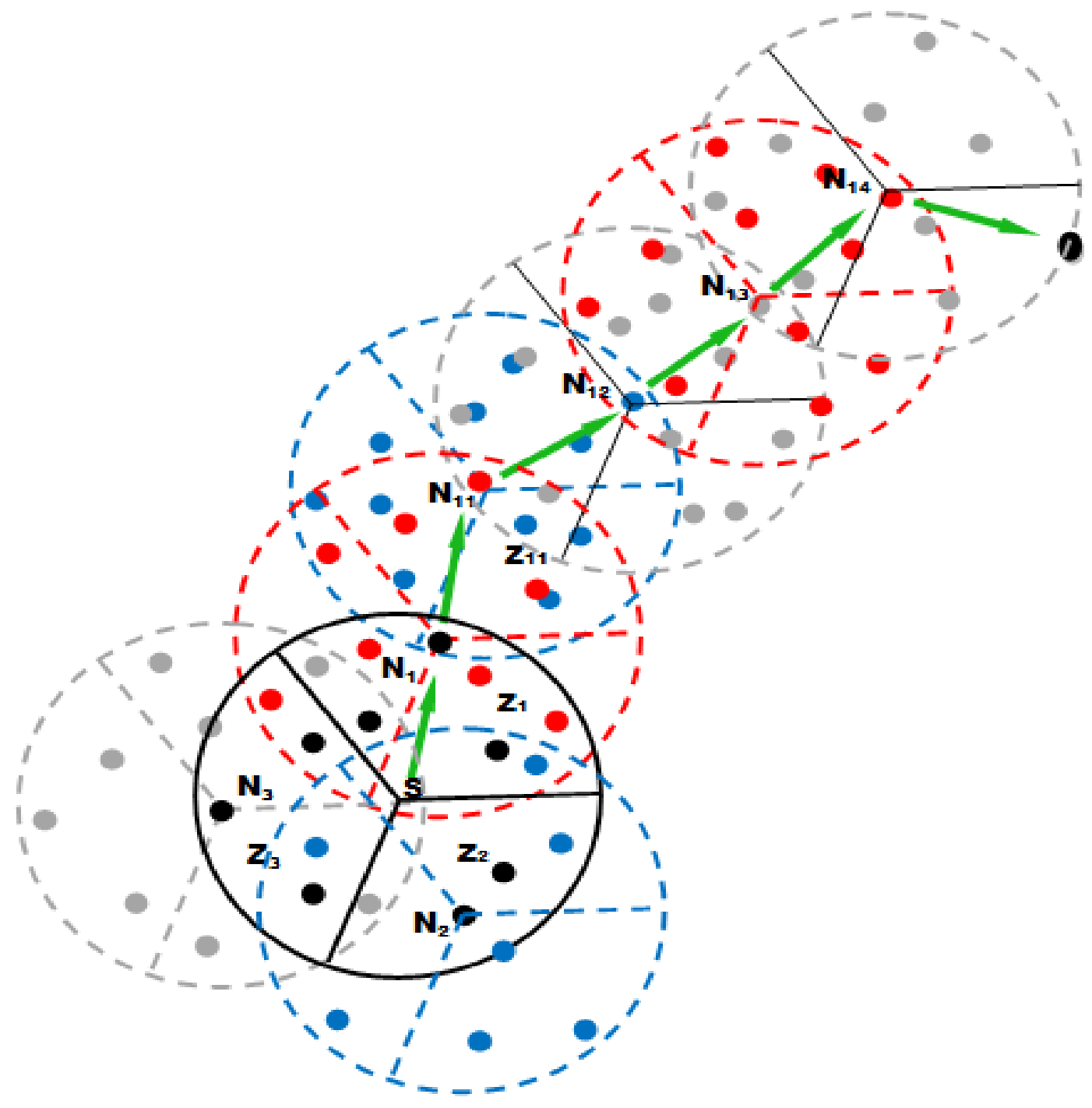

Fig 4: Path maintenance of PEA

Using ADOV protocol the minimum hop from which node in the zone is determined. The figure 4 shows that in first zone set the node $\mathrm{N}_{1}$ because ADOV determine the minimum hop count is $\mathrm{N}_{1}$ to $\mathrm{D}$. comparison other node hop distance. Then the packet sent to the $\mathrm{N}_{1}$. After receiving the packet $\mathrm{N}_{1}$ find the Maximum distance node in his three zones. After compression got the node of each zone is $\mathrm{N}_{11}, \mathrm{~N}_{12}, \mathrm{~N}_{13}$. Now using flooding concept determined the destination node and hop count to $\left(\mathrm{N}_{11}, \mathrm{~N}_{12}, \mathrm{~N}_{13}\right.$ to D). Here got the minimum hop $\mathrm{N}_{11}$ to $\mathrm{D}$. so packet is send to the $\mathrm{N}_{11}$ using this concept Maintenance the path. Here a big advantage is that for node mobility destination node moves anywhere. Using this concept each timestamp it check the destination. so here packet lost is minimum and maintenance the path without lass overhead.

\section{PATH ESTABLISHMENT AND MAINTENANCE ALGORITHM}

Input: number of nodes present in each zone (n), transmitting frequency range (freq), wavelength for the frequency (Wlen) and the packet processing time for each node which is constant Max distance( int $\mathrm{n}$, float freq, float BT, float when, float Tp)
S1- for $\mathrm{i}:=1$ to $\mathrm{n}$

$\mathrm{i}:=\mathrm{i}+1$

Input Receiving Time RT[i] of a node

Calculate Transceiver Time TT[i] : = RT[i] - BT $-2 \mathrm{Tp}$

Calculate Distance D[i] : = wlen $\times$ freq $\times$ TT $[\mathrm{i}]$

for $\mathrm{j}:=\mathrm{j}$ to 32

$\mathrm{j}:=\mathrm{j}+1$

IP address of a node in a zone IP $[\mathrm{i}, \mathrm{j}]:=$ IP address of RT[i]

S2- Initialized max distance : $=\mathrm{D}[1]$

S3- for $\mathrm{j}=1$ upto 32

$\mathrm{j}:=\mathrm{j}+1$

$\operatorname{IP} 1[\mathrm{j}]:=\operatorname{IP}[1, \mathrm{j}]$

S4- 


$$
\begin{aligned}
& \mathrm{i}:=\mathrm{i}+1 \\
& \text { if max distance }<D[i+1] \\
& \text { then } \\
& \text { max distance }:=\mathrm{D}[\mathrm{i}+1] \\
& \text { for } \mathrm{j}:=1 \text { upto } 32 \\
& \quad \mathrm{j}:=\mathrm{j}+1 \\
& \quad \mathrm{IP} 1[\mathrm{j}]:=\operatorname{IP}[\mathrm{i}, \mathrm{j}]
\end{aligned}
$$

S5- $\quad$ Return value IP1

S6- $\quad \operatorname{IPX}[\mathrm{i}]=\mathrm{IP}[\mathrm{I}, \mathrm{j}]$

Return IPX[I] as maximum distance node

S7- $\quad D[i]:=$ distance between IP $[\mathrm{i}, \mathrm{j}]$ from destination node

$$
\mathrm{D}[\mathrm{i}]=\mathrm{L}[\mathrm{h}][\mathrm{x}]
$$

$\mathrm{L}[\mathrm{h}][\mathrm{x}]:=$ no of hop to reach destination node

S8-

$$
\text { initialize } L[h][x]=L[1][x]
$$

$$
\text { Calculate } \mathrm{L}[\mathrm{h}][\mathrm{x}]
$$

If $\mathrm{L}[\mathrm{h}][\mathrm{x}]$ is minimum

$$
\text { Set D[h]: = as D[i] }
$$

*It's run three times for $\mathrm{L}[\mathrm{h}][1], \mathrm{L}[\mathrm{h}][2], \mathrm{L}[\mathrm{h}][3]$

S9- $\quad$ set X[i] node as next node

S10- call S1 to S5 for X[i] node

\section{PERFORMANCE ANALYSIS}

Here present the results of Path Establishment and Maintenance Algorithm. The results from our simulations were collated into several statistics files and subsequent data analysis was undertaken by analyzing these files. For the simulation of mobile networks, the results are divided into three main parts. The first part shows the End to End Delay of Path Establishment and Maintenance Algorithm.. The second part is Throughput achieved by Path Establishment and Maintenance Algorithm. The ratio of packets delivered against packets generated by the Application layer is used as the throughput measure. This is important as any claims to improving the routing algorithm must be backed up by an increase in performance in this area. The last part deals with the packet loss of Path Establishment and Maintenance Algorithm. The packet loss of this algorithm significantly low than others in a MANETs.

\subsection{Simulation Setup}

For the simulation of the algorithm, latest version 2.34 of NS-2 has been used.. Ns-2.32 is a discrete event simulator targeted at networking research.

\section{Scenario}

Topology of 500*500 is taken for simulation.

Nodes are being generated randomly at random position.

Nodes are generated at random time as if few nodes are entering into the topology.

Nodes are moving at constant random speed.
Radio propagation model used is Two-Ray Ground. Antenna model used is Omni Antenna

Movement is linear and node speed is constant for a simulation

\section{Node Characteristics:}

Link Layer Type: Logical Link (LL) type

MAC type: 802_11

Queue type: Drop-Tail

Network Interface type: wireless

Channel type: wireless

Table 1. The simulation parameters of Path Establishment and Maintenance Algorithm

\begin{tabular}{|c|c|}
\hline Parameter & Value \\
\hline Simulator & NS-2 (Version 2.34 ) \\
\hline $\begin{array}{c}\text { Radio-propagation } \\
\text { model }\end{array}$ & Propagation/Two ray round wave \\
\hline $\begin{array}{c}\text { Network interface } \\
\text { type }\end{array}$ & Wireless \\
\hline MAC Type \\
\hline $\begin{array}{c}\text { Interface queue } \\
\text { Type }\end{array}$ & Queue/Drop Tail \\
\hline Link Layer Type & LL \\
\hline Antenna \\
\hline $\begin{array}{c}\text { Maximum packet } \\
\text { in ifq }\end{array}$ & Antenna/Omni Antenna \\
\hline Area (X*Y) & 200 \\
\hline $\begin{array}{c}\text { Number of mobile } \\
\text { node }\end{array}$ & 500 \\
\hline Source Type & 30 \\
\hline Simulation Time & UDP \\
\hline Routing Protocols & $60 \mathrm{~s}$ \\
\hline
\end{tabular}

\subsection{Performance Metrics}

The following performance metrics are evaluated to understand the behavior routing Path Establishment and Maintenance Algorithm .

The average end to end delay.

Throughput

Packet Loss

\section{SIMULATION RESULTS}

After simulation the graph is plotted for four different numbers of receiving nodes $1,6,4$, and 16 when the packet size 512 byte, rate $600 \mathrm{~kb}$, and maximum no of packets is 1000 . At the start of simulation, all the nodes are laid out randomly on the square area and then start moving towards a random destination at some random speed 0 to maximum specified speed. After reaching the destination the nodes start to move towards the next randomly chosen destination. In this simulation graph, path between 10 to 1 represent in Red colour, path between 3 to 6 represent in Green colour, path between 20 to 4 represent in Blue colour and path between 25 to 16 represent in Yellow colour. 

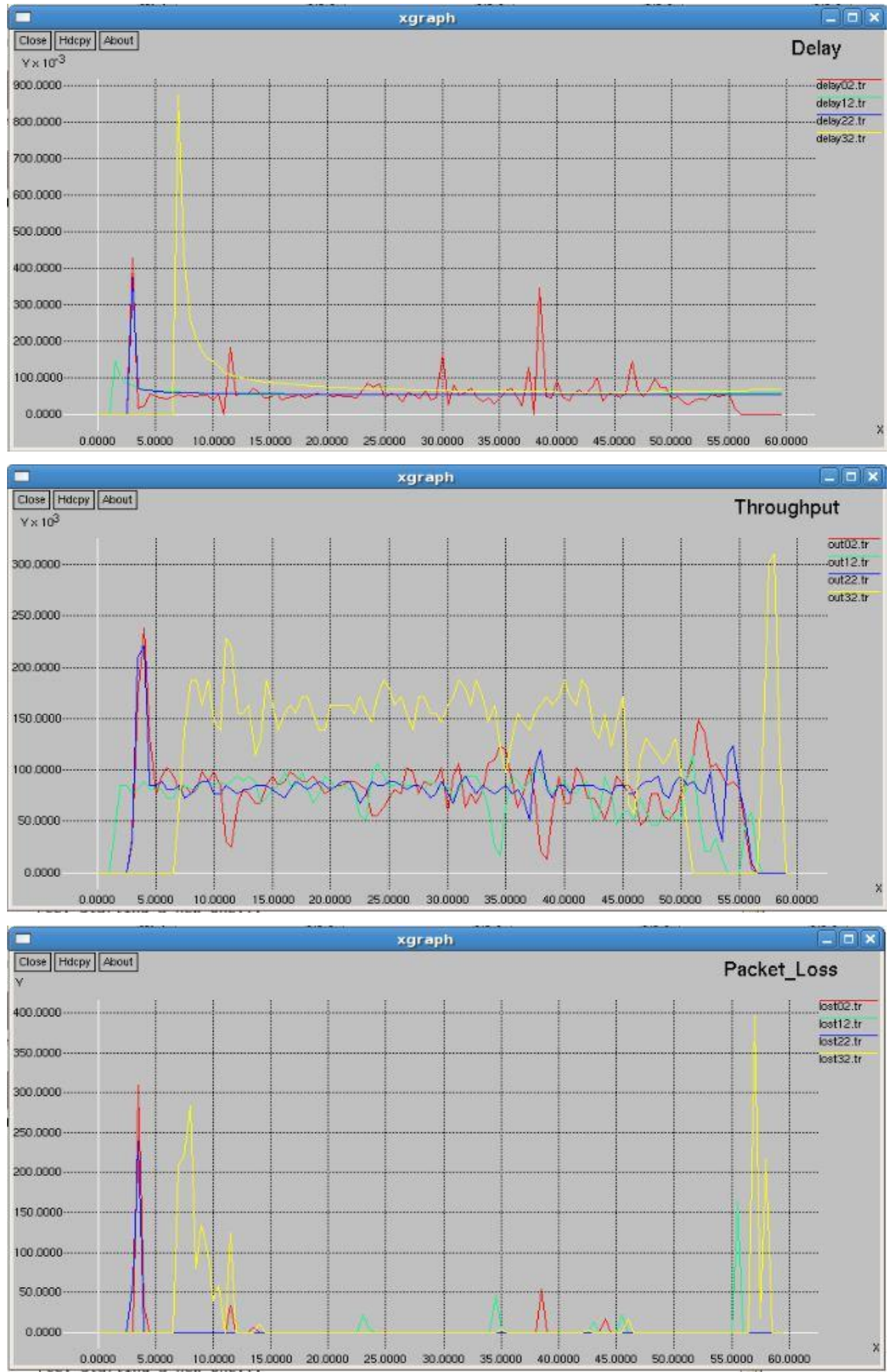

Fig 5: (a) End to End delay (b) Throughput (c) Packet loss of PEA

\section{CONCLUSION}

In this paper we proposed Path Establishment Maintenance Algorithm which is analyzed by exploiting a very important ad hoc network characteristic of multiple paths between nodes. The performance of Path Establishment and Maintenance Algorithm is less packet drop, good performance in Throughput and end to end delay to other protocols. Optimization schemes are applied so that a forwarding node and its neighbors can collaborate to adapt the path to both topology change and traffic demand and thus improve transmission path opportunistically. Path 
Establishment and Maintenance Algorithm can be combined with other protocol which considers other aspects than confidentiality to improve significantly the efficiency of security systems in ad hoc networks.

\section{REFERENCES}

[1]. Abolhasan M, Wysocki T, Dutkiewicz E 'A review of routing protocols for mobile ad hoc networks' Ad Hoc Networks 2, 2004 pp 1-22.

[2]. Agarwal U, Yadav K. P., Parik K, Nithesh K., Nandakumar "Routing optimization in mobile ad-hoc networks using proactive and reactive protocols" International Journal of Research Review in Engineering Science and Technology ,2012 Volume-1 Issue-2 (ISSN 2278- 6643)

[3]. Anuj G K, Harsh S, Anil V K "Performance analysis of AODV, DSR \& TORA Routing Protocols", IACSIT International Journal of Engineering and Technology, Vol.2, No.2. 2010

[4]. Basu S D, Charu W, Shalini S "Comparison Of Proactive And Reactive Routing Protocols In Mobile Adhoc Network Using Routing Protocol Property”, International Journal of Emerging Technology and Advanced Engineering, 2012 Volume 2, Issue 3.

[5]. Dhenakaran S S, Parvathavarthini A“ An Overview of Routing Protocols in Mobile Ad-Hoc Network", Parvathavarthini et al., International Journal of Advanced Research in Computer Science and Software Engg 3(2),2013 pp. 251-259

[6]. Gupta A K , Sadawarti S and Verma A K "Performance analysis of AODV, DSR \& TORA Routing Protocols" IACSIT International Journal of Engineering and Technology, 2010 ,Vol.2, No.2, ISSN: 1793-8236.

[7]. Jose Moses G, Kumar S, Varma P.Suresh, and Supriya N "A Simulation Based Study of AODV, DSR, DSDV Routing Protocols in MANET Using NS-2" International Journal of Advanced Research in Computer Science and
Software Engineering, 2012 Volume 2, Issue 3, ISSN: 2277 128X.

[8]. Kargar M, Fartash F, Saderi T, M. Dishabi M Ebrahimi “A Theory in Optimization of Ad-hoc Routing Algorithms" World Academy of Science, Engineering and Technology 242008 .

[9]. Kumar M P, Gaurav S, Vaishali S, Ashish R, Srivastava S M ," An Overview of AODV Routing Protocol”, 2012, Proceedings Vol.2, Issue.pp-728-732, ISSN: 2249-664.

[10]. Maurya P K, Sharma G, Sahu V, Roberts A, and Srivastava A "An Overview of AODV Routing Protocol" International Journal of Modern Engineering Research (IJMER) 2012, Vol.2, Issue.3, pp-728-732 ISSN: 2249-6645

[11].Rajeshkumar V, Sivakumar P (2013) “Comparative Study of AODV, DSDV and DSR Routing Protocols in MANET Using Network Simulator-2" International Journal of Advanced Research in Computer and Communication Engineering Vol. 2, Issue 12, ISSN (Print) 2319-5940 ISSN (Online) : 2278-1021

[12].Santra S and A P Pinaki ," Comparative Study of Proactive Routing Protocols for MANETs" International Journal of Electronics and Computer Science Engineering ISSN: 2277-1956/V2N1-375-384 Volume2, Number 1 page 375 to 383 .

[13].Santra, S. and Maji, P. 'Path Establishment Algorithm for Mobile Ad Hoc Network : A novel approach' santanu santra et.al , int.j. computer technology \& applications, 2013, vol 4 (3),450-455.

[14]. Shivahare ,B. Wahi, C. and, Shivhare, S. 'Comparison Of Proactive And Reactive Routing Protocols In Mobile Adhoc Network Using Routing Protocol Property:' International Journal of Emerging Technology and Advanced Engineering, 2012 (ISSN 2250-2459, Vol 2, Issue 3). 